\title{
Opciones de gestión agronómica para la variabilidad y para el cambio climático: El riego localizado ${ }^{1}$
}

\author{
Lincoln Zotarelli, Clyde Fraisse, and Daniel Dourte ${ }^{2}$
}

Esta serie de publicaciones EDIS proporciona información sobre diferentes opciones de gestión agronómica disponibles para mejorar la eficiencia del uso de los recursos, la adaptación a la variación y al cambio climático. Para consultar la serie completa de estas publicaciones, visite http://edis.ifas.ufl.edu/ topic_series_agricultural_management_options.

\section{Introducción}

La adaptación a la variabilidad y al cambio climático se puede lograr a través de una amplia gama de alternativas de gestión y de avances tecnológicos.

Mientras que la toma de decisiones en la agricultura involucra otros aspectos que van más allá del clima, incluyendo la economía, factores sociales y las consideraciones de las política, los riesgos relacionados al clima son la fuente principal del rendimiento y de la variabilidad en los ingresos. Las estrategias existentes pueden ayudar a los productores a minimizar los riesgos asociados con la variabilidad y el cambio climático también como mejorar la eficiencia del uso de los recursos existentes en la finca. Esta serie de publicaciones EDIS ofrece información sobre las tecnologías existentes, y esta publicación se enfoca en el uso del riego localizado para mejorar los sistemas de producción.

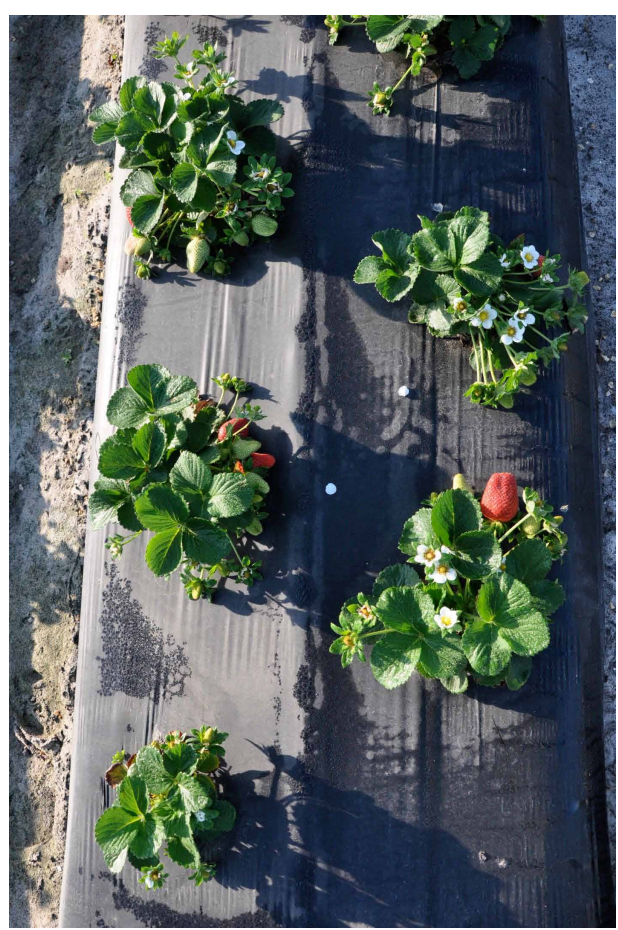

Figure 1. Riego por goteo aplicado a la superficie para la producción de fresas. Créditos: Lincoln Zotarelli

\section{¿Qué es el riego localizado?}

El riego localizado es la aplicación lenta y frecuente de agua directamente en un área relativamente pequeña adyacente a plantas individuales a través de emisores colocados a lo largo de la línea de suministro de agua. El agua es

1. Este documento, HS1212, es uno de una serie de publicaciones del Horticultural Sciences, Servicio de Extensión Cooperativa de la Florida, Instituto de Alimentos y Ciencias Agrícolas, Universidad de la Florida. (UF/IFAS). Fecha de primera publicación: January 2013. Visite nuestro sitio web EDIS en $<$ http://edis.ifas.ufl.edu>. This publication is a translation of Agricultural Management Options for Climate Variability and Change: Microirrigation.

2. Lincoln Zotarelli, assistant professor, Horticultural Sciences Department; Clyde Fraisse, assistant professor, and Daniel Dourte, postdoctoral research associate, Agricultural and Biological Engineering Department, University of Florida Institute of Food and Agricultural Sciences, Gainesville, FL 32611. 
generalmente transportada a baja presión en un tubo plástico flexible. Generalmente, el agua debe ser de alta calidad para evitar la obstrucción de los pequeños emisores, lo que es a menudo manejado con la filtración y con el tratamiento ocasional de químicos. Una ventaja principal en el riego localizado es que la evaporación no beneficiosa, en otras palabras, la evaporación del agua de la superficie de los suelos y de las cubiertas vegetales que no contribuye con el crecimiento de las plantas - se reduce considerablemente cuando se compara con el riego por aspersión. El riego localizado es un término amplio que incluye varios métodos de aplicación.

- Riego por goteo: El agua se aplica a la superficie del suelo a través de pequeños emisores, ubicados usualmente junto o cerca de la planta a ser irrigada (Figuras 1 y 2). El emisor de índice bajo usualmente es de 3 galones por hora, y el índice de aplicación (pulgadas/día) depende del emisor y del espacio lateral.

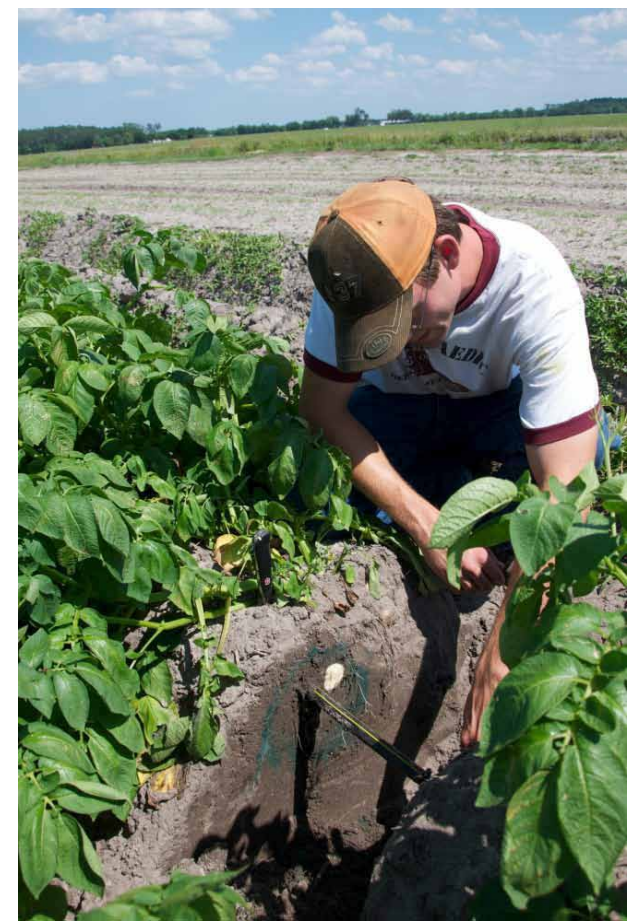

Figure 2. Riego por goteo subterráneo (SDI) para las patatas en Hastings, Florida. Créditos: Lincoln Zotarelli

- El riego por goteo subterráneo (SDI): El agua se aplica por debajo de la superficie del suelo por medio de las líneas de goteo laterales que se instalan a una profundidad de 12-18 pulgadas (Figura 3). La labranza, la implantación del cultivo y las otras operaciones sobre el terreno no son impedidas por los laterales porque estos han sido establecidos a una profundidad suficiente para permitir las operaciones en el terreno y el uso a largo plazo. El flujo de agua de SDI es generalmente menos de 3 galones por hora. SDI puede tener una vida útil de hasta 20 años, lo que le convierte en el método más económicamente competitivo junto al riego por pivote central en sistemas de cultivo por hilera (Lamm et al. 2010).

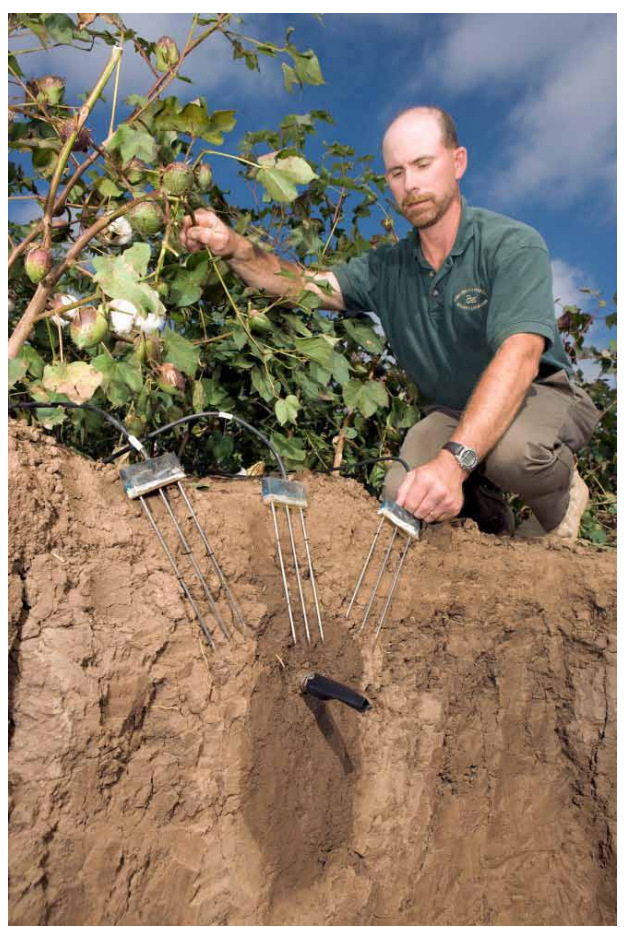

Figure 3. SDI colocado profundamente en un campo de algodón permite una larga vida útil y evita la interferencia con la labranza. El uso de sondas de monitoreo permite la medición del agua en el suelo. Créditos: Peggy Greb

- El riego micropulverizado: El agua se aplica a la superficie del suelo en forma de aerosol o rocío por medio de emisor colocado de 6-12 pulgadas sobre la superficie del suelo. Los índices de aplicación son usualmente menores a 40 galones por hora. El riego pulverizado puede proporcionar el servicio adicional de protección contra los daños por congelación en algunos cultivos hortícolas.

\section{¿Cómo el riego localizado reduce los riesgos relacionados con el clima?}

Cualquier tipo de riego en un sistema de agricultura comercial puede reducir en grandes medidas los riesgos relacionados con el clima, que resultan del estrés hídrico (Harwood et al. 1999). Debido a una eficiencia mejorada y una presión de plagas reducida, el riego localizado puede proporcionar alguna medida adicional en reducir el riesgo en comparación con los sistemas de riego por aspersión. Esto puede ser resumido de la siguiente manera: 
- Debido a su alta eficiencia (menor evaporación de agua en la superficie del suelo, menor deriva del viento y menor evaporación de agua interceptada por el follaje), el riego localizado reduce el volumen de agua necesaria para producir los cultivos, lo cual puede reducir el riesgo de escasez de suministro de agua para el riego.

- El riego localizado permite la flexibilidad en el momento y la cantidad de agua a aplicar, de acuerdo a la evapotranspiración/demanda por la planta.

- Debido a que menos cantidad de agua es aplicada, la lixiviación de los nutrientes es reducida.

- La aplicación de los nutrientes puede ser mejor programada para satisfacer las necesidades de la plantas. La aplicación de los fertilizantes al agua de riego significa que los nutrientes pueden ser entregados directamente a la zona radicular.

- El riego localizado permite el uso de cobertura de polietileno, lo que ayuda a conservar la humedad del suelo y reducir la lixiviación de fertilizantes por la precipitación.

- El riego localizado puede ser usado para proteger los pequeños cultivos hortícolas de las heladas.

\section{¿Cuáles son los beneficios agronómicos?}

La humectación reducida de la superficie del suelo y del dosel de las plantas puede resultar en una reducción de la presión de enfermedades y malezas. Mejores rendimientos de algunos cultivos en hileras de bajo costo, irrigados con SDI en comparación con el equipo de pivote central, han sido demostrados. Consulte el ejemplo de algodón en la Figura 4. Además, un bajo impacto en el consumo de agua (la proporción en uso de agua/cosecha obtenida) se ha observado en el de SDI, incluso cuando se compara con las tecnologías de alta eficiencia en pivote centrales (Figura 5). La siguiente lista es un resumen de los beneficios agronómicos más destacados del riego localizado:

- Reducción del consumo del agua: Debido a que el riego por goteo trae el agua a la zona de las raíces sin mojar el campo completo, el riego por goteo típicamente requiere $25 \%-50 \%$ del volumen de agua necesitado por similares sistemas de riego por aspersión (Lamm y Trooien 2003).

- Reducción de los problemas de plagas: la maleza y los problemas de enfermedades pueden reducirse debido a que el riego por goteo no moja el medio entre las hileras o el follaje de los cultivos como el riego por aspersión

- Reducción de la compactación superficial: El riego localizado reduce la formación de una corteza en la superficie del suelo, que puede ocurrir debido a la repetición de aplicaciones de rocío.

- Manejo conjunto de riego y fertilización: el riego por goteo puede mejorar la eficiencia de las aplicaciones de agua y del fertilizante. La aplicación precisa de los nutrientes es posible usando el riego por goteo, potencialmente reduciendo los costos de los fertilizantes y las pérdidas de nutrientes solubles.

- Ventajas de producción: En sistemas hortícolas, cuando se combinan con levantamiento de camas, cubierta de polietileno y trasplantes, el riego por goteo aumenta el rebrote temprano y uniformidad de los cultivos. Usando la cubierta de polietileno también aumenta la limpieza de los productos cosechados y reduce el riesgo de la contaminación con agentes patógenos del suelo. Coberturas reflectantes también ayudan a reducir la incidencia de enfermedades virales afectando a los insectos portadores, tales como lostrips, moscas blancas o pulgones.

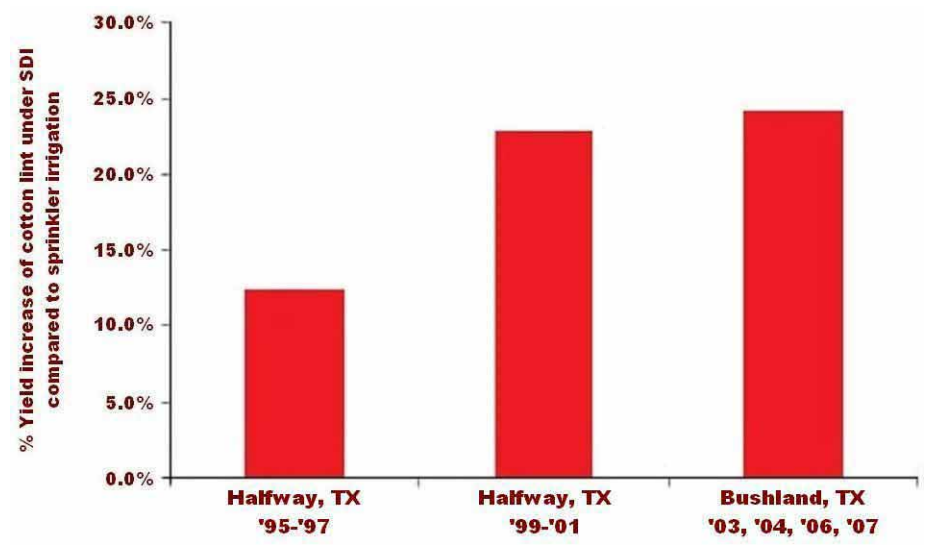

Figure 4. El aumento de los rendimientos de la fibra de algodón por el SDI en comparación con el sistema por aspersión en estudios en Halfway, Texas, y Bushland, Texas, reportados por Lamm et al. 2010. Los aumentos en el rendimiento de algodón por SDI fueron atribuidos a una mayor proporción del agua de riego contribuyendo a la transpiración (menos evaporación del suelo) y un ambiente más cálido resultante de la ausencia de humedecimiento del suelo con SDI. Créditos: Daniel Dourte

\section{¿Cuáles son los impactos en los costos de producción?}

- Las necesidades de menos bombeo: El sistema de goteo requiere una baja presión para la operación (20-25 psi en la entrada del campo, 10-12 psi en la cinta de goteo) 


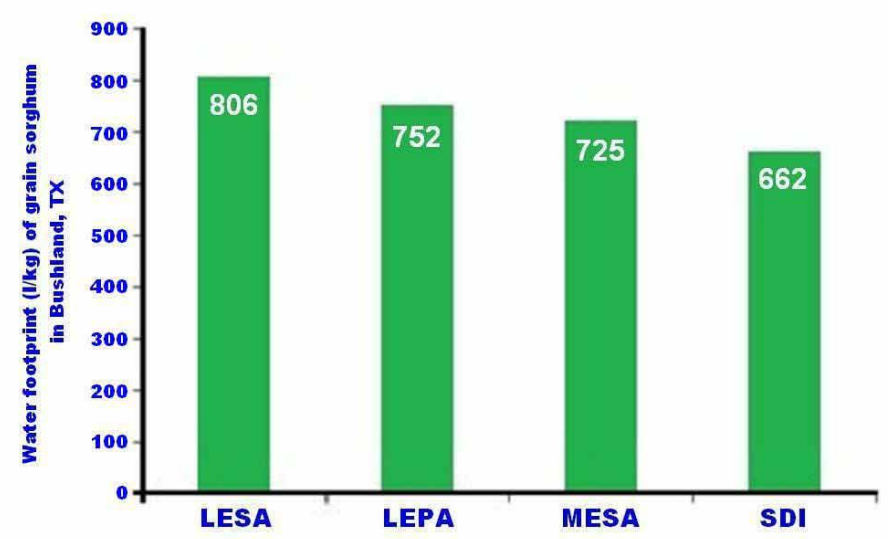

Figure 5. Un impacto significativamente menor de agua en el sorgo con SDI en comparación con el riego por aspersión fue observado durante las temporadas del 2000-2002 en Bushland, Texas (Colaizzi et al. 2004). Créditos: Daniel Dourte

en comparación con los sistemas por aspersión (50-80 psi). Muchas de las pequeñas bombas y los pozos pueden utilizarse para regar adecuadamente un terreno de pocos acres usando sistemas de goteo.

- Automatización: La aplicación de riego por goteo puede ser simplemente manejada y programada usando controladores de riego, reduciendo así lo costos laborales.

- Flexibilidad: Los sistemas de goteo son adaptables a campos de forma irregular o aquellos con una topografía o textura de suelo irregular, eliminando así las esquinas no utilizadas o sin cultivos y maximizando el uso del terreno disponible.

\section{¿Cuál es el costo de la inversión?}

Los sistemas de riego por goteo suelen costar alrededor de $\$ 500-\$ 1,500$ por acre. Una parte del costo es una inversión de capital útil por varios años, y la otra parte es debido al costo anual de las piezas desechables. Los productores que comienzan con el riego por goteo deberían empezar con un sistema simple en un terreno de pocos acres antes de moverse a un sistema más grande. El SDI para los cultivos en hileras se ha encontrado menos costoso que el sistema de riego con pivote central en un campo de maíz de 160-acres para un SDI con una vida útil mayor de 15 años (Lamm et al. 2012).

\section{¿Cuáles son los impactos de las emisiones de gases del efecto invernadero?}

El SDI ha demostrado que resulta en una disminución sustancial de las emisiones de $\mathrm{N}_{2} \mathrm{O}$ en comparación con las emisiones del riego en surco (Sánchez-Martin et al. 2008). Los costos del combustible de SDI en comparación con el riego con pivote central pueden ser de \$5-\$15 menos por acre para el riego de maíz (O’Brien et al. 1997; Lamm et al. 2012), sugiriendo que reducciones relacionadas con la energía y emisiones de $\mathrm{CO}_{2}$ pueden ser observadas con el SDI.

\section{¿Cuáles son las barreras y los incentivos para la implementación?}

\section{Barreras}

- La inversión económica

- La falta de información sobre el sistema, su manejo correcto y mantenimiento

- Agua de alta calidad

\section{Incentivos}

- Los programas de participación de costo para la conservación de agua - Se espera que los productores calificados contribuyan con una porción del costo total del proyecto.

- Los programas de conservación de agua administrados por el USDA (Por ejemplo, El Programa de Incentivos de calidad ambiental [EQIP])

- Los programas de conservación de agua regional y estatal, por ejemplo: BMP el Programa de Participación de Costo [FDASC]; el Programa FARMS [SWFWMD]; El Programa de Participación de Costo para la Protección de Agua Sostenible [SJRWMD])

\section{Agradecimientos}

Esta información fue desarrollada en contribución con el proyecto "Variabilidad del Clima al Cambio de Clima: Retos de Extensión y Oportunidades en el Sudeste de E.E.U.U." y fue auspiciado por la Iniciativa Competitiva de investigaciones de Alimentos y Agricultura no. 2011-6700330347 del instituto de Agricultura y Alimentos del USDA

\section{Referencias}

Colaizzi, P. D., A. D. Schneider, S. R. Evett, and T. A. Howell. 2004. "Comparison of SDI, LEPA, and Spray Irrigation Performance for Grain Sorghum." Trans. ASAE 47 (5): 1477-1492. 
Harwood, J., R. G. Heifner, K. Coble, P. Janet, and A. Somwaru. 1999. Managing Risk in Farming: Concepts, Research and Analysis. Washington, DC: Economic Research Service, USDA.

Lamm, F. R., P. D. Colaizzi, J. P. Bordovsky, T. P. Trooien, J. Enciso-Medina, D. O. Porter, D. H. Rogers, and D. M. O’Brien. 2010. “Can Subsurface Drip Irrigation (SDI) Be a Competitive Irrigation System in the Great Plains Region for Commodity Crops?" 2010. Proceedings of the 5th National Decennial Irrigation meeting of the ASABE, Phoenix Convention Center, 5-8 December 2010, Phoenix, AZ USA.

Lamm, F. R., D. M. O’Brien, D. H. Rogers, and T. J. Dumler. 2012. "Using the K-State Center Pivot Sprinkler and SDI Economic Comparison Spreadsheet." Proceedings of the 24th Annual Central Plains Irrigation Conference, Colby, Kansas, February 21-22. http://www.ksre.ksu.edu/sdi/ Reports/2012/LammUsingCPSDI12.pdf.

Lamm, F. R., and T. P. Trooien. 2003. "Subsurface Drip Irrigation for Corn Productivity: A Review of 10 Years of Research in Kansas." Irrig. Sci. 22 (3-4): 195-200.

O’Brien, D. M., D. H. Rogers, F. R. Lamm, and G. Clark. 1997. "Economic Comparison of SDI and Center Pivots for Various Field Sizes." Kansas State University. http://www. ksre.ksu.edu/library/ageng2/mf2242.pdf.

Sanchez-Martin, L., A. Arce, A. Benito, L. Garcia-Torres, and A. Vallejo. 2008. "Influence of Drip and Furrow Irrigation Systems on Nitrogen Oxide Emissions from a Horticultural Crop." Soil Biology \& Biochemistry 40 (7): 1698-1706.

\section{Información Adicional}

Clark, G. A., and D. Z. Haman. 2011. Microirrigation in Mulched Bed Production Systems: Irrigation Depths. AE72. Gainesville: University of Florida Institute of Food and Agricultural Sciences. http://edis.ifas.ufl.edu/ae049.

Florida Agricultural Water Conservation Best ManagementPractices. [2012.] http://www.floridaagwaterpolicy.com/.

Haman, D. Z. 2011. Scheduling Tips for Drip Irrigation of Vegetables. AE259. Gainesville: University of Florida Institute of Food and Agricultural Sciences. http://edis.ifas. ufl.edu/ae092.

Haman, D. Z., and F. T. Izuno. 2003. Principles of Microirrigation. AE70. Gainesville: University of Florida Institute of Food and Agricultural Sciences. http://edis.ifas.ufl.edu/ wi007.

Haman, D. Z., and A. G. Smajstrla. 2010. Design Tips for Drip Irrigation of Vegetables. AE260. Gainesville: University of Florida Institute of Food and Agricultural Sciences. http://edis.ifas.ufl.edu/ae093.

Simonne, E., R. Hochmuth, J. Breman, W. Lamont, D. Treadwell, and A. Gazula. 2012. Drip-Irrigation Systems for Small Conventional Vegetable Farms and Organic Vegetable Farms. HS1144. Gainesville: University of Florida Institute of Food and Agricultural Sciences. http://edis.ifas.ufl.edu/ hs388. 